World Conference on Marine Biodiversity - Montreal

THEME: STRATEGIES FOR CONSERVATION OF MARINE BIODIVERSITY Session 1: Biodiversity restoration

Carlo Cerrano ${ }^{1}$, Tatjana Bakran-Petricioli ${ }^{2}$, Joana Boavida ${ }^{3}$, Eliana Ferretti ${ }^{4}$, Andrea Gari ${ }^{5}$, Daniel Gomez-Gras $^{6}$, Andrea Gori ${ }^{6}$, Bernat Hereu ${ }^{5}$, Silvija Kipson ${ }^{2}$, Martina Milanese ${ }^{4}$, Jean-Baptiste Ledoux $^{6}$, Cristina Linares ${ }^{5}$, Àngel López-Sanz ${ }^{6}$, Paula López-Sendino ${ }^{6}$, Ignasi Montero-Serra ${ }^{5}$, Marta Pagès ${ }^{5}$, Daniela Pica ${ }^{1}$, Antonio Sarà ${ }^{4}$, Ester Serrao ${ }^{3}$, Fabrizio Torsani ${ }^{1}$, Núria Viladrich ${ }^{6}$, Joaquim Garrabou ${ }^{6}$

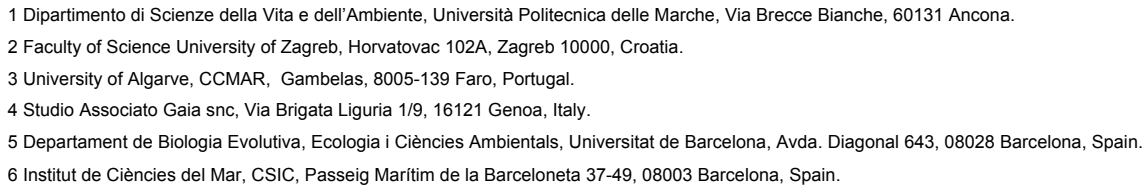

Corresponding author:

Eliana Ferretti

e.ferretti@studioassociatogaia.com

\title{
Restoring biodiversity in the Mediterranean coralligenous - the MERCES project
}

The temperate coralligenous bioconcretions are mainly built by the accumulation of encrusting coralline algae growing at low irradiance levels. They harbour approximately $10 \%$ of marine Mediterranean species (about 1600 species), including long-lived algae and invertebrates. Enhanced by climate change, several pressures affect coralligenous assemblages, leading to increased frequency of mass mortalities and dramatic loss of habitat complexity and biodiversity. The EU-funded project MERCES is developing innovative methodologies to restore macroinvertebrate habitat-forming species from three key taxonomic groups: Cnidaria/Anthozoa, Porifera/Demospongiae and Bryozoa. Restoration protocols are based on fragments/transplants from donor organisms and recruitment-enhancing devices. Collaboration with volunteers (divers and diving operators) in several phases of field activities proved crucial both to minimize underwater working time and to increase the sense of stewardship in a major users' segment. Additionally, the MERCES project explores how to enhance restoration success by identifying the mechanisms conferring resistance to thermal stress in gorgonians. This is pursued by coupling thermotolerance experiments with next generation sequencing tools and facilitation processes (by comparing the outcomes of mono-specific vs. multi-specific transplanted assemblages). Results will be included in the strategic planning of restoration efforts in the Mediterranean in the context of global change.

Keywords: Restoration, coralligenous, Mediterranean, key species

Short description: The EU-funded project MERCES is developing restoration approaches for the Mediterranean coralligenous 